\title{
Accelerating Anisotropic Cosmological Model Filled with Two Fluid and $\Lambda$
}

\author{
S. M. Borikar
}

\begin{abstract}
In this paper we have studied Kantowski-Sachs universe filled with perfect fluid and radiation with a cosmological constant. To get determinate solution, it is assumed that the scalar of expansion is proportional to shear scalar $(\theta \alpha \sigma)$ which leads to the relation between metric potentials $R=A S^{n}$ The cosmological parameters of models are also discussed.

Keywords: Perfect fluid, Radiation, cosmological constant, Kantowski-Sachs space-time.
\end{abstract}

\section{INTRODUCTION}

The spatially homogeneous and anisotropic Kantowski-Sachs model has astro-physically important because they are considered as possible candidates for an early era in cosmology. The recent observations indicate that the universe at large scale is homogeneous and isotropic (Knop et.al (2003), Gasprini (2003), Riess et.al. (2004)) and is in accelerating phase. Exact solutions of general theory of relativity for homogeneous space-time belong to either Bianchi types or Kantowski-Sachs (Roy Choudhari (1979)).

Kantowski-Sachs models have been studied by Weber (1984, 85), Lorcaz (1983), Gron (1986), Matravers (1988), Krori et.al.(1995), Li \& Hao (2003). Sing and Agrawal discussed Kantowski-Sachs model in Seaz Ballester (1985) and scalar tensor theory (1991). Pradhan and Yadav (2002) investigated Kantowski-Sachs model with variable G and . Adhav et.al. (2011) studied Kantowski-Sachs model with anisotropic dark energy.

To understand the early and present stages of the universe the spatially homogeneous and isotropic models like FRW are considered as good approximation. Many authors investigated FRW models with two fluid source (Davidson 1962; McIntosh 1968). Two fluid models where one fluid is the radiation field corresponding to the observed cosmic background radiation, while the matter content of the universe is represented by perfect fluid are studied.( Coley and Tupper 1986; Coley 1988). An interacting two fluid FRW universe is investigated by Pradhan et.al. (2011). Two fluid cosmological models in Bianchi space-time have been studied by Coley and Dunn (1990), Pant and Oli (2002), Oli

Revised Manuscript Received on February 05, 2020.

* Correspondence Author

S. M. Borikar, Department of Applied Mathematics, SIPNA College of Engineering and Technology, Amravati, India. Email: soniyanishant@gmail.com

(C) The Authors. Published by Blue Eyes Intelligence Engineering and Sciences Publication (BEIESP). This is an open access article under the CC BY-NC-ND license (http://creativecommons.org/licenses/by-nc-nd/4.0/)
(2008), Adhav et.al. (2011).

In this paper we assume that the geometry of the universe is that of a Kantowski-Sachs space-time. An analytic solution of general relativistic field equations is presented and physical behavior of the corresponding non-interacting fluids model is discussed.

\section{METRIC AND FIELD EQUATIONS}

We consider the Kantowski-Sachs space-time in the form

$$
d s^{2}=d t^{2}-R^{2} d r^{2}-S^{2}\left(d \theta^{2}+\sin ^{2} \theta d \phi^{2}\right)
$$

where $R$ and $S$ are functions of time ' $t$ ' only.

The Einstein field equations are (with gravitational units, $8 \pi G=1, c=1$ ) given by

$$
R_{i}^{j}-\frac{1}{2} \delta_{i}^{j} R+\Lambda=-\left(T_{i}^{j(m)}+T_{i}^{j(r)}\right)
$$

where $R_{i}^{j}$ is the Ricci tensor, $R$ is the Ricci scalar and $T_{i}^{j(m)}$ and $T_{i}^{j(r)}$ are the energy-momentum tensor for matter and radiation field respectively and $\Lambda$ is a cosmological constant.

The energy-momentum tensors for matter (perfect fluid) and radiation are given by

$$
\begin{aligned}
& T_{i}^{j(m)}=\left(\rho_{m}+p_{m}\right) u_{i} u^{j}-p_{m}, \\
& T_{i}^{j(r)}=4 / 3 \rho_{r} u_{i} u^{j}-1 / 3 \rho_{r} ;
\end{aligned}
$$

with components

$$
\begin{array}{ll}
T_{1}^{1(m)}=T_{2}^{2(m)}=T_{3}^{3(m)}=-p_{m} & , T_{0}^{0(m)}=\rho_{m} \\
T_{1}^{1(r)}=T_{2}^{2(r)}=T_{3}^{3(r)}=-\rho_{r} / 3 & , T_{0}^{0(r)}=\rho_{r}
\end{array}
$$

Where $\quad p_{m}, \rho_{m}$ and $\rho_{r}$ are pressure and densities of matter and radiation respectively.

Einstein's field equations for line element (1) using equations (2) and (4) are

$2 \frac{\ddot{S}}{S}+\frac{\dot{S}^{2}}{S^{2}}+\frac{1}{S^{2}}+\Lambda=-p_{m}-\frac{\rho_{r}}{3}$,
$\frac{\ddot{R}}{R}+\frac{\ddot{S}}{S}+\frac{\dot{R} \dot{S}}{R S}+\Lambda=-p_{m}-\frac{\rho_{r}}{3}$,

$$
\frac{2 \dot{R} \dot{S}}{R S}+\frac{\dot{S}^{2}}{S^{2}}+\frac{1}{S^{2}}-\Lambda=\rho_{m}+\rho_{r} \text {, }
$$




\section{Accelerating Anisotropic Cosmological Model Filled with two Fluid And $\Lambda$}

Where overhead dots denotes derivatives with respect to time ' $t$ '. The three independent equations (5) - (7) connect five unknown variables $\left(R, S, p_{m}, \rho_{m}, \rho_{r}\right)$. Thus, to obtain a determinate solution, we assume supplementary condition $\theta \alpha \sigma$, which leads to the relation

$$
R=A S^{n}
$$

Where $A$ and $n$ both are constants.

We also assume the relation between pressure and density of matter field through the 'gamma law' equation of state as

$$
p_{m}=(\gamma-1) \rho_{m}, \quad 1 \leq \gamma<4 / 3
$$

Using (8) and (9), the field equations (5) - (7) reduces to

$$
\begin{aligned}
& 2 \frac{\ddot{S}}{S}+\frac{\dot{S}^{2}}{S^{2}}+\frac{1}{S^{2}}+\Lambda=-(\gamma-1) \rho_{m}-\frac{\rho_{r}}{3}, \\
& (n+1) \frac{\ddot{S}}{S}+n^{2} \frac{\dot{S}^{2}}{S^{2}}+\Lambda=-(\gamma-1) \rho_{m}-\frac{\rho_{r}}{3}, \\
& (2 n+1) \frac{\dot{S}^{2}}{S^{2}}+\frac{1}{S^{2}}-\Lambda=\rho_{m}+\rho_{r} .
\end{aligned}
$$

Subtracting equation (11) from (10), we get

$$
\ddot{S}+(n+1) \frac{\dot{S}^{2}}{S}+\frac{1}{1-n} \frac{1}{S}=0
$$

To solve equation (13), we denote $\dot{S}=\eta$ and $\ddot{S}=\eta \frac{d \eta}{d s}$.

Using above substitutions (13) reduces to first order differential equation

$$
\eta \frac{d \eta}{d s}+(1+n) \frac{\eta^{2}}{S}+\frac{1}{1-n} \frac{1}{S}=0
$$

Solving equation (14), we obtain

$$
\eta=\left[\frac{1}{n^{2}-1}+c S^{-2(1+n)}\right]^{1 / 2}
$$

Where $c$ is constant of integration.

Hence the line element (1) reduces to

$$
d s^{2}=\left[\frac{1}{n^{2}-1}+c S^{-2(1+n)}\right]^{-1} d s^{2}-A^{2} S^{2 n} d r^{2}-S^{2}\left(d \theta^{2}+\sin ^{2} \theta d \phi^{2}\right)
$$

Under suitable transformation of coordinates, equation (16) becomes

$$
d s^{2}=\left[\frac{1}{n^{2}-1}+c T^{-2(1+n)}\right]^{-1} d T^{2}-A^{2} T^{2 n} d r^{2}-T^{2}\left(d \theta^{2}+\sin ^{2} \theta d \phi^{2}\right)
$$

The physical and geometrical parameters for the above model are obtained. The expression for densities of matter $\rho_{m}$ and radiation $\rho_{r}$, the scalar of expansion $\theta$, the shear scalar $\sigma^{2}$ , the deceleration parameter $q$ and spatial volume $V$ are given by $\rho_{m}=\frac{1}{4-3 \gamma}\left[\frac{-2 c(1+2 n)}{T^{2(n+2)}}+\frac{2\left(n^{2}+n+1\right)}{\left(n^{2}-1\right) T^{2}}\right]+2 \Lambda$,

$\rho_{r}=\frac{3 c(2-\gamma)(2 n+1)}{(4-3 \gamma) T^{2(n+2)}}+\frac{6 n(1-\gamma)+n^{2}(2-3 \gamma)-2}{(4-3 \gamma)\left(n^{2}-1\right) T^{2}}+\Lambda$

$p_{m}=\frac{\gamma-1}{(4-3 \gamma)}\left[\frac{2\left(n^{2}+n+1\right)}{\left(n^{2}-1\right) T^{2}}-\frac{2 c(1+2 n)}{T^{2(n+2)}}\right]$,

$$
\theta=\frac{n+2}{T}\left[\frac{1}{n^{2}-1}+c T^{-2(1+n)}\right]^{1 / 2} \text {, }
$$

$\sigma^{2}=\frac{2(n-1)}{3(1+n) T^{2}}+\frac{2 c(1-n)^{2}}{3 T^{2(2+n)}}$,

$q=\frac{3 c(n+1) T^{-2(1+n)}}{(n+2)\left[\frac{1}{n^{2}-1}+c T^{-2(1+n)}\right]}-\frac{n-1}{n+2} ; n \neq-2$

$V=A T^{n+2}$,

$\Delta=\frac{1}{3} \sum_{i=1}^{3}\left(\frac{H_{i}-H}{H}\right)^{2}=2\left(\frac{n-1}{n+2}\right)^{2}$.

\section{DISCUSSION}

It is observed that both the energy densities for matter and radiation are positive, provided $n>1$. The densities $\left(\rho_{m}\right.$ and $\rho_{r}$ ) and the scalar of expansion ( $\theta$ ) tends to infinity as $T \rightarrow 0$. Whereas $\rho_{m}$ and $\rho_{r}$ tends to finite when $T \rightarrow \infty$. Hence the model represents the shearing and non-rotating expanding universe with a Big-Bang start. For $n=1$, the shear scalar $\sigma$ is zero. Therefore $n=1$ is the isotropy condition. The spatial volume tends to zero as $T \rightarrow 0$ and $V \rightarrow \infty$ as $T \rightarrow \infty$.i.e. volume starts with zero and increases with increase in $T$ for $n>0$. The model accelerate or decelerate depends on the value of $n$. The deceleration parameter is zero for $n=1$ which indicates the universe is expanding with uniform velocity. It is observed that model does not exists for $n=1$.

Also, the model does not approach isotropy for large values of $T$ because $\lim _{T \rightarrow \infty} \frac{\sigma}{\theta} \neq 0$. The value of the anisotropic parameter of the expansion is constant that means the anisotropy is maintained throughout the end of the universe. 


\section{SPECIFIC MODELS}

Table- II: Anisotropic Parameter

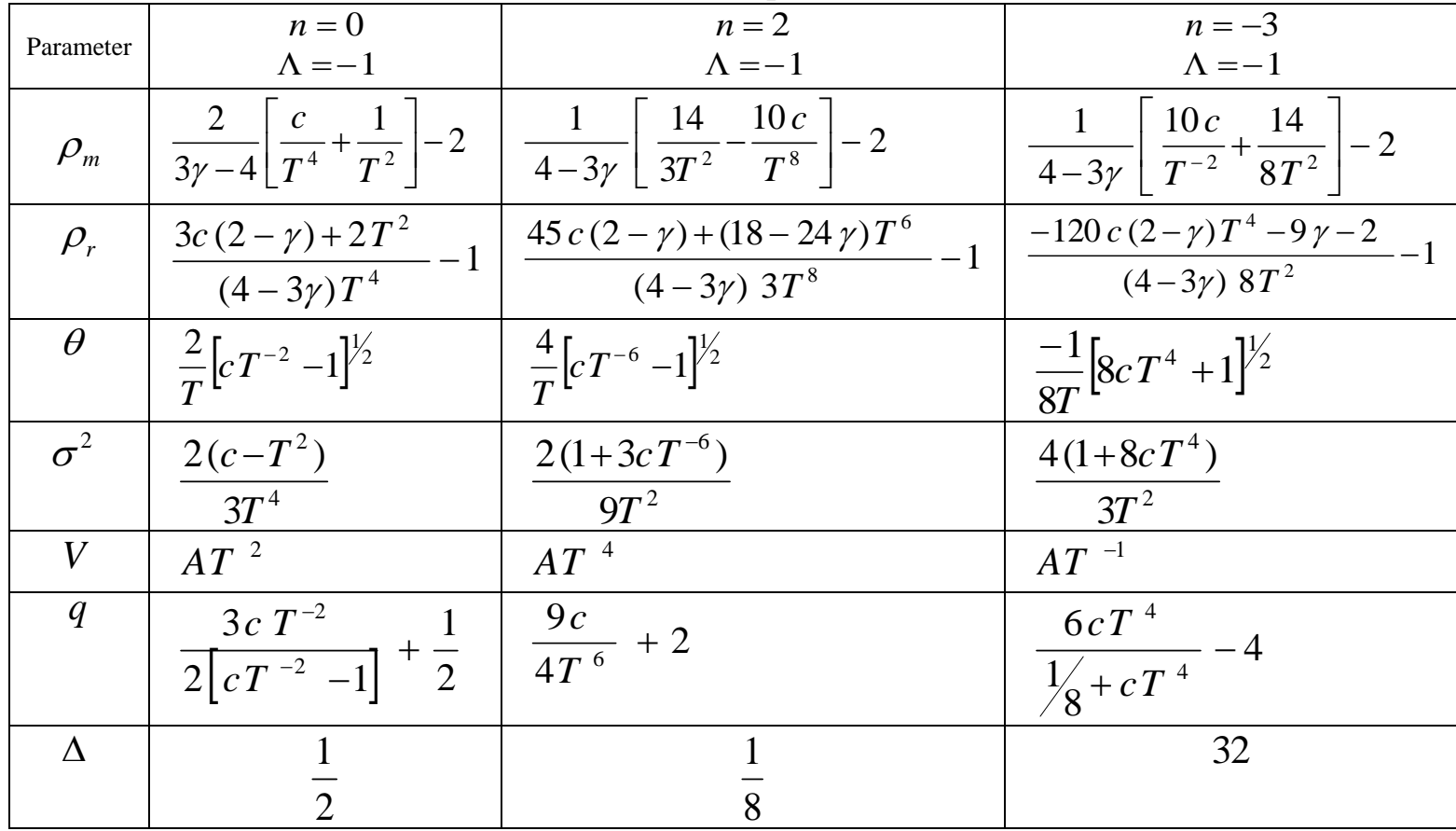

In the above table we have given matter density, radiation density, the scalar of expansion, the shear scalar, spatial volume, deceleration parameter and the anisotropic parameter of the expansion for different values of $n$.

\section{CONCLUSION}

We have investigated the system of two interacting and non-interacting fluids and $\Lambda$. The comparative study of above models shows that $n=2$ gives satisfactory results for $c=1$ and $1 \leq \gamma<4 / 3$. More precise value of $\Lambda$ from recent observations will give standard model of the universe.
25. Weber : Journal of Mathe. Phys. 26, 1308 (1985).

\section{AUTHORS PROFILE}

Dr. S. M. Borikar completed her Ph.D.in Mathematics from Sant Gadge Baba Amravat University, Amravati. Currently working as Assistant Professor at Sipna College of Engineering and Technology, Amravati. She has presented papers at various National and international conferences both home and abroad, published articles and papers in various international journals. Her main area of interest is in relativity.

\section{REFERENCES}

1. Adhav et. al.: Cont.Eur.J.Phys. 9(4), 919-925 (2011).

2. Adhav et.al.: EJTP ,25,1-8 (2011).

3. Coley A.A., Dunn K.: Astrophys.J.348, 26(1990).

4. Coley A.A., Tupper B.O.J.: J.Math. Phys. 27,406(1986).

5. Coley A.A.: Astrophys. Space Sci.140, 175 (1988).

6. Davidson W.: Mon. Not. R. Astron. Soc. 124, 79 (1962).

7. Gasprini M.,et.al.: Phys Rept.373,1-212 (2003).

8. Gron : Journal of Mathe. Phys. 32, 2456 (1986).

9. Knop et.al.: The Astrophysical J., 598,102 (2003).

10. Krori et.al.: Journal of Mathe. Phys., 36,991-1553 (1995).

11. Li , Hao: arXiv: astro-ph/0308288 (2003).

12. Lorenz D.: Astrophysics and Space Sci, 98, 101-113 (1984).

13. Matravers D.R.: Gen Rel. and Gravitation, 20,279 (1988).

14. McIntosh, C.B.G.:Mon. Not. R. Astron.Soc.140,461(1968).

15. Pant D.N.,Oli S.: Astrophys, Space Sci.281,623(2002).

16. Pant D.N.,Oli S.: Astrophys, Space Sci.281,623(2002).

17. Pradhan A., Amirhashchi H. \& Saha B. : Astrophys.Space Sci. 33,1,(2011).

18. Pradhan and Yadav: Int. J. Mod. Phys. D 11, 893 (2002).

19. Riess A.G.:et al.: Astophys. J.607, 665 (2004).

20. Roy Choudhari, A.K.: Theoretical cosmology, Oxford, Clarendon Press (1979).

21. Sanjay Oli : Astrophys Space Sci 314, 89-94 (2008).

22. Sanjay Oli : Astrophys Space Sci 314, 95-103 (2008).

23. Sing T., Agrawal A.: Astrophys Space Sci., 182, 289-312 (1991).

24. Weber : Journal of Mathe. Phys. 25, 3279 (1984). 